\title{
Absolute and Relative Deprivation and the Measurement of Poverty
}

\author{
by
}

Jean-Yves Duclos

Department of Economics and CRÉFA, Université Laval, Canada and UNSW, Sydney, Australia

and

Philippe Grégoire

CRÉFA, Université Laval, Canada

and Department of Economics, University of Western Ontario, Canada

\begin{abstract}
This paper develops the link between poverty and inequality by focussing on a class of poverty indices (some of them well-known) which aggregate normative concerns for absolute and relative deprivation. The indices are distinguished by a parameter that captures the ethical sensitivity of poverty measurement to "exclusion" or "relative-deprivation" aversion. We also show how the indices can be readily used to predict the impact of growth on poverty. An illustration using LIS data finds that the United States show more relative deprivation than Denmark and Belgium whatever the percentiles considered, but that overall deprivation comparisons of the four countries considered will generally necessarily depend on the intensity of the ethical concern for relative deprivation. The impact of growth on poverty is also seen to depend on the presence of and on the attention granted to concerns over relative deprivation.
\end{abstract}

Keywords Poverty, relative deprivation, inequality, poverty alleviation.

JEL numbers D6, I3, O1.

This research was supported, in part, by grants from the Social Sciences and Humanities Research Council of Canada and from the Fonds FCAR of the Province of Québec. The hospitality of the Institut d'Anàlisi Econòmica (CSIC) at the Universitat Autònoma de Barcelona (where this paper was revised) is also gratefully acknowledged. We further wish to thank Michael Hoy, Gordon Anderson and Kuan Xu for their useful comments.

Corresponding address: Jean-Yves Duclos, Département d'économique, Pavillon de Sève, Université Laval, Québec, Canada, G1K 7P4; Tel.: (418) 656-7096; Fax: (418) 656-7798; Email: jduc@ecn.ulaval.ca 


\section{Introduction}

Since the work of Sen (1976), taking into account inequality among the poor, and not solely the incidence or average intensity of poverty, has become common scientific practice and has generated a considerable literature 1. Alongside this has grown a belief among several researchers and policy analysts that concerns of relativity are also important in assessing poverty lines. In the words of Townsend (1979), a well-known proponent of that relativist view:

"Individuals, families and groups in the population can be said to be in poverty when they lack the resources to obtain the type of diet, participate in the activities and have the living conditions and amenities which are customary, or are at least widely encouraged or approved, in the societies to which they belong. Their resources are so seriously below those commanded by the average individual or family that they are, in effect, excluded from ordinary living patterns, customs and activities." (p.31)

The link between poverty and relative exclusion from society also transpires from the official use of the concept of social exclusion in the European Commission, where it is defined "in relation to the social rights of citizens (...) to participation in the major social and occupational opportunities of the society." (Room (1992), p.14) On his part, Sen believes that comparing poverty across distributions may involve "different standards of minimum necessities" (1981, p.21) and "that absolute deprivation in terms of a person's capabilities relates to relative deprivation in terms of commodities, incomes and resources" (1984, p.326). This view is somewhat supported by the large number of cross-country comparisons using proportions of median or mean incomes as poverty lines.

Another link between poverty and relativity is the frequent normalisation of poverty indices by possibly different poverty lines (see, for instance, Foster et al. (1984)), which typically leads to "relative poverty indices" as defined in Blackorby and Donaldson (1980). Foster and Shorrocks (1988), Foster and Sen (1997) and Davidson and Duclos (2000) show how such normalisation links relative poverty and relative inequality comparisons. Finally, having identified the poor and measured the respective intensity of their poverty, individual poverty is usually aggregated into global poverty indices, and 'in the 'aggregation' exercise the magnitudes of absolute depri-

\footnotetext{
${ }^{1}$ See e.g., Takayama (1979), Kakwani (1980), Clark, Hemming and Ulph (1981), Atkinson (1987) and Foster et al. (1984) for such work, and Foster (1984), Chakravarty (1990), Foster and Sen (1997) and Zheng (1997), among others, for a review of different aspects of the social welfare, poverty, and inequality literatures.
} 
vation may have to be supplemented by considerations of relative deprivation" (Sen (1981),p.32).

Among all these links between poverty, inequality and exclusion, it is on the one between poverty and relative deprivation in the latter "aggregation exercise" that we wish to focus particularly in this paper ${ }^{2}$. We will do this by developing a class of poverty indices which combine concerns of absolute deprivation and of relative deprivation. Absolute deprivation is undoubtedly "an irreducible core (...) in our idea of poverty, which translates reports of starvation, malnutrition and visible hardship onto a diagnosis of poverty" (Sen (1981), p.17). Although sometimes neglected by economists, relative deprivation has been linked to "definable and measurable social and psychological reactions, such as different types of alienation" (Durant and Christian (1990), p.210) by social psychologists and to social protests, discrimination, feelings of injustice and subjective ill-being (Olson (1986)). It has also been used to interpret measures of inequality and income redistribution (see for instance Yitzhaki (1979) and Duclos (2000)).

The class of poverty indices we consider in this paper is a generalisation of the Sen(1976)-Thon(1979)- Chakravarty(1983)-Shorrocks(1995) indices of poverty. The indices $S(v)$ depend upon an ethical parameter $v$ which captures the sensitivity of poverty measurement to "exclusion" or "relativedeprivation" aversion. The greater the value of $v$, the greater the weight assigned to relative deprivation as against absolute deprivation in measuring and comparing poverty.

The next section sets up the basic definitions and shows the link between generalised Gini indices and relative deprivation, upon which our subsequent work draws. Section 2 then shows how our class of poverty indices can be understood as a weighted sum of absolute and relative deprivation. It further points to the indices' useful and simple graphical interpretation as weighted areas underneath cumulative poverty gap (CPG) curves, and indicates how they can be used to assess the impact of growth on poverty and for decomposition analyses. Section 2 also compares the properties of the $S(v)$ indices with those of additive poverty indices (most saliently, with the popular class of FGT indices (Foster, Greer and Thorbecke (1984))).

Section 3 illustrates some of the results using Luxembourg Income Study data drawn from 4 countries. For a reasonable common poverty line, we find

\footnotetext{
${ }^{2}$ For this aggregative exercise, an absolute or a relative poverty line can be equally well be used. For what follows, however, we assume this line to be the same for the measurement of absolute and relative deprivation. The aggregation exercise and the results of the paper could, however, be extended to the use of different poverty lines for the measurement and the aggregation of absolute and relative deprivation.
} 
that, whatever the percentiles considered, the United States have more relative deprivation than Denmark and Belgium, but that the relative deprivation curve for Italy crosses that of the three other countries. Moreover, for all but one of the six possible country comparisons, it is not possible to make unambiguous robust poverty orderings based on CPG curves. Since absolute deprivation and mean poverty are very similar in the four countries, we thus find that unambiguous poverty comparisons would inevitably depend on the importance granted to concerns over relative deprivation. The impact of growth on poverty is also seen to depend on the presence of and on concerns for relative deprivation: in pairwise comparisons, poverty is least responsive to growth in the USA and in Denmark, which is also where relative deprivation is the greatest. The last section concludes our paper.

\section{Inequality and relative deprivation}

Consider the cumulative distribution of income $F$ with support contained in the nonnegative real line. Let a poverty line be denoted by $z$, and define the headcount index as $H=F(z)$. Denote by $y^{*}(p)$ the quantile function associated to $F \cdot y^{*}(p)$ is formally defined as $y^{*}(p)=\inf \{s \geq 0 \mid F(s) \geq p\}$ for $p \in[0,1]$. For a continuous and strictly increasing distribution, $y^{*}(p)$ is simply $F^{-1}(p)$ and can be thought of as the income of the individual whose rank (or percentile) is $p$. Let $y(p)$ be the income $y^{*}(p)$ when censored at $z$, with $y(p)=\min \left(y^{*}(p), z\right)$, and denote the poverty gap of an individual at rank $p$ by $g(p)=(z-y(p))$. Note therefore that $g(p)=0$ for $p \geq H .{ }^{3}$

The next most popular poverty index after the headcount is traditionally denoted by $H I$, the average poverty gap in the population:

$$
H I=\int_{0}^{1} g(p) d p .
$$

\footnotetext{
${ }^{3}$ Note here that we have not normalised poverty gaps by the poverty line. This normalisation would make no substantial difference whenever the poverty lines are the same across all distributions being compared. The normalisation will in fact be desirable if poverty lines are designed to act as price indices in order to transform nominal incomes into real incomes (making living standards comparable across distributions with different prices). It is not clear, however, that such a normalsation is an appropriate procedure when poverty lines differ for reasons other than differences in prices (see, e.g., Atkinson (1991) and Davidson and Duclos (2000)). For instance, it might be that differences in climatic conditions or normative judgements set a higher poverty line in real terms in some distributions than in others. Normalising poverty gaps by the respective poverty lines would then push the analysis away from comparing absolute deprivation towards comparing deprivation and poverty gaps as a proportion of different poverty lines, a feature which could potentially lead to invalid rankings of well-being and deprivation across the distributions.
} 
Hence, if perfect targetting of the poor were possible, $H I$ would give the per capita expenditures which the state would need to spend in order to eradicate poverty completely. Clearly, and as we will discuss more later, $H I$ does not give any ethical or normative weight to inequality in the distribution of the poverty gaps.

Let the cumulative poverty gap (CPG) curve be defined as:

$$
G(p)=\int_{0}^{p} g(s) d s .
$$

This curve was introduced by Jenkins and Lambert (1997), who called it a "TIP" curve, and subsequently by Shorrocks (1998), who labelled it a "Poverty Profile" curve (see also on this the work by Spencer and Fisher (1992)). It is clear from (2) that :

$$
\frac{d G(p)}{d p}=g(p)
$$

By definition, we have that $G(0)=0$ and $G(p)=H I$ for $p \geq H$. $G(p)$ thus becomes saturated at $p=H . G(p) / p$ is the average poverty gap of the $100 \cdot p \%$ poorest members of the population. $G(H) / H$ is the average poverty gap of the poor, often defined in the literature (see Sen (1976)) as $I$. As we shall see, the curvature of the CPG curve also shows the extent of inequality in the distribution of the poverty gaps.

The CPG curve is continuous, non-decreasing and concave in $p$, as we can see on Figure 1, where CPG curves $G_{A}(p)$ and $G_{B}(p)$ have been drawn for two hypothetical distributions, $A$ and $B$. As can be seen on the Figure, $A$ has everywhere a greater cumulative poverty gap whatever the percentage of the poorest part of the population considered. $A$ has also more inequality among its poor than $B$ (for which all poor have the same incomes, as can be seen from the initial straight line segment). $A$ has nevertheless a lower headcount than $B$. In determining which of $A$ or $B$ has more poverty, there may therefore exist a trade-off between the number of the poor (the "incidence" of poverty $H$ ), the overall average poverty gap (the average "intensity", $H I$ ), and the inequality in poverty (the curvature of $G(p)$ ).

The class of poverty indices $S(v)$ on which we will focus in this paper will all indicate that poverty is greater in $A$ than in $B$ (although the headcount index clearly would not). This is because $G_{A}(p)$ is everywhere greater than $G_{B}(p)$. This ordering of poverty in terms of CPG curves is in fact valid for a broader class of poverty indices than $S(v)$, as shown in Jenkins and 
Lambert (1997) and in Shorrocks (1998). Let $\Pi$ be the class of poverty indices $\pi$ that are replication invariant, increasing and Schur-convex in $g(p)$. Then,

$$
G_{A}(p) \geq G_{B}(p) \forall p \in[0,1] \text { if and only if } \pi_{A} \geq \pi_{B} \forall \pi \in \Pi .
$$

A useful tool for capturing the inequality in the distribution of poverty gaps is the Lorenz curve of the distribution of censored incomes, defined as $L(p)=\frac{1}{\mu} \int_{0}^{p} y(s) d s . \mu$ is the mean of the distribution of censored incomes; with equation (1) and $g(p)=z-y(p)$, this gives $H I=z-\mu$. This allows a decomposition of the CPG curve into components due to the mean and to the inequality of poverty gaps:

$$
\begin{aligned}
G(p) & =\int_{0}^{p} z-y(s) d s \\
& =p(z-\mu)+\mu(p-L(p)) \\
& =\underbrace{p \cdot H I}_{A}+\underbrace{\mu(p-L(p))}_{B}
\end{aligned}
$$

where

$A \equiv$ poverty of the $100 \cdot p \%$ poorest if aggregate poverty HI were equally distributed across the population

$B \equiv$ excess poverty for the $100 \cdot p \%$ poorest due to the inequality in the distribution of aggregate poverty.

Hence, the value of $G(p)$ can be split in two parts, mean deprivation (A) and "excess" deprivation due to inequality of poverty (B), as shown in Figure 2. As Figure 2 also suggests, we will see later that this decomposition gives rise respectively to absolute and relative deprivation.

To capture inequality of poverty in an aggregate index, first recall that the Gini index of inequality is given by ${ }^{4}$ :

$$
I=2 \int_{0}^{1}(p-L(p)) d p .
$$

The Gini index is thus the average distance between population shares and income shares of all possible proportions (between 0 and 1 ) of the poorer in a population. A well-known single-parameter generalisation of the Gini

\footnotetext{
${ }^{4}$ This and subsequent definitions are given implicitly for distributions of censored incomes, but they can clearly apply to any distribution of living standards.
} 
(or "s-Gini" 5 ) is obtained by applying the normative weights $k(p, v)=$ $v(v-1)(1-p)^{v-2}$, for $v \geq 1$, to the distance $p-L(p)$ between the line of perfect equality and the Lorenz curve:

$$
I(v)=\int_{0}^{1}(p-L(p)) k(p, v) d p .
$$

For $v=1$, no weight is attached to inequality, and $I(1)=0$. For $1<v<2$, $k(p, v)$ increases with $p$, and thus greater weight is attributed to the distance $p-L(p)$ at larger proportions of the population. For $v=2$, the weight is equal to 2 everywhere, and $I(2)$ is thus the standard Gini coefficient defined in (8). For $v>2$, the weight given to the distance $p-L(p)$ between population share and income share decreases with $p$, and more and more rapidly so as $v$ rises. Note that $k(p, v)$ (for integers $v>1$ ) can be interpreted as the probability that an individual with rank $p$ in the population finds himself the poorest among $v-1$ individuals randomly selected from the population (see, e.g., Muliere and Scarsini (1989), Lambert (1993) and Duclos (2000)). Now define :

$$
\omega(p, v)=\int_{p}^{1} k(s, v) d s=v(1-p)^{v-1}
$$

By integration by parts, we can show that the index $I(v)$ in (9) equals ${ }^{6}$

$$
I(v)=\int_{0}^{1} \omega(p, v) \frac{\mu-y(p)}{\mu} d p
$$

It is well-known that the standard Gini coefficient can be understood as an index of relative deprivation (Sen (1973), Yitzhaki (1979) and Hey and Lambert (1980)). Duclos (2000) also shows a similar result for the s-Gini. To see this, assume that an individual $i$ with rank $p_{i}$ feels the following relative deprivation $\delta\left(p_{i}, p_{j}\right)$ when he compares himself to an individual $j$ with rank $p_{j}$ :

\footnotetext{
${ }^{5}$ See Kakwani (1980), Donaldson and Weymark $(1980,1983)$ and Yitzhaki (1983).

${ }^{6}$ For expositional simplicity, the derivation of equations (11), (14) and (15) is shown in the appendix. For ease of reference, also note that in a discrete setting with a finite population of $n$ individuals, the weight on an individual with rank $j, j=1, \ldots, n$, (when individuals are sorted in increasing values of incomes) equals (see Donaldson and Weymark (1980)):

$$
\omega(j / n, v)=\frac{1}{n^{v}}\left((n-j+1)^{v}-(n-j)^{v}\right)
$$
}




$$
\delta\left(p_{i}, p_{j}\right)=\left\{\begin{array}{cc}
y\left(p_{j}\right)-y\left(p_{i}\right) & \text { if } p_{j}>p_{i} \\
0 & \text { otherwise. }
\end{array}\right.
$$

This formulation has often been justified by reference to the classical definition of relative deprivation found in Runciman (1966, p.10): "The magnitude of a relative deprivation is the extent of the difference between the desired situation [e.g., the income of the richer] and that of the person desiring it". Note again here that we use censored income instead of just income.

The expected relative deprivation of individual $i$ with respect to the whole population of $j$ 's is then given by

$$
c\left(p_{i}\right)=\int_{0}^{1} \delta\left(p_{i}, p\right) d p
$$

Combining (12) and (13) yields:

$$
c\left(p_{i}\right)=\mu\left(1-L\left(p_{i}\right)\right)-\left(1-p_{i}\right) y\left(p_{i}\right) .
$$

We now wish to aggregate each individual's relative deprivation into an overall index. To do this, we may take an ethically weighted mean of $c(p)$, with weights equal to $k(p, v)$. We can then show that:

$$
I(v)=\frac{1}{v \mu} \int_{0}^{1} c(p) k(p, v) d p .
$$

The standard Gini coefficient is thus obtained as a mean-normalised expected relative deprivation in the population :

$$
G(2)=\frac{1}{2 \mu} \int_{0}^{1} c(p) d p
$$

More generally, for integers $v>1$, the s-Gini $I(v)$ is the expected relative deprivation of the individual who finds himself the most deprived out of a group of $v-1$ individuals randomly drawn from a population. Thus, the greater the value of $v$, the more weight is given to the relative deprivation of the poorer (see Duclos (2000) for more on this). 


\section{A Class of Poverty Indices}

\subsection{Poverty and Deprivation}

Now define a single-parameter index of poverty $S(v)$ (along the lines of the $I(v)$ index $^{7}$ ) as a weighted area underneath the CPG curve $G(p)$ :

$$
S(v)=\int_{0}^{1} k(p, v) G(p) d p .
$$

By integration by parts, proceeding as for (11), we can show that $S(v)$ can also be expressed as a weighted sum of poverty gaps, with the weights equal to $\omega(p, v)$ :

$$
S(v)=\int_{0}^{1} \omega(p, v) g(p) d p .
$$

(18) can be understood as a special case of a more general class of linear poverty measures, in the spirit of Mehran (1976) for inequality indices and Yaari (1988) for social welfare indices. From equations (6) and (17), note that

$$
S(v)=\int_{0}^{1} k(p, v)(p \cdot H I) d p+\int_{0}^{1} k(p, v) \mu(p-L(p)) d p
$$

and that $S(v)$ has therefore a nice graphical interpretation in Figure 2 as the sum of the weighted area of absolute deprivation and of the weighted area showing inequality in censored incomes. By equations (9), (15) and (19), we also obtain the immediate result that the $S(v)$ index is a sum of expected absolute and relative deprivation in the distribution of censored incomes :

$$
\begin{aligned}
S(v) & =\int_{0}^{1} k(p, v) p \cdot H I d p+\mu I(v) \\
& =\underbrace{H I}_{A}+\underbrace{\frac{1}{v} \int_{0}^{1} k(p, v) c(p) d p}_{B}
\end{aligned}
$$

where

$A \equiv$ average absolute deprivation (average shortfall from the poverty line)

$B \equiv$ average relative deprivation (weighted sum of $c(p)$ ).

\footnotetext{
${ }^{7}$ The link between $S(v)$ and the s-Gini indices of inequality is briefly mentioned in Chakravarty (1983, p.81). For other references to that class of poverty indices, see Hagenaars (1987) and Shorrocks (1998).
} 
The $S(v)$ indices are thus an ethically weighted sum of absolute and relative deprivation. Absolute deprivation is the average shortfall (HI) from the poverty line. Relative deprivation is the ethically weighted average shortfall from the incomes of others. Note that these comparison incomes are censored at the poverty line. This censoring of reference incomes at the poverty line can be justified by the view of Runciman (1966, p.29) that "people often choose reference groups closer to their actual circumstances than those which might be forced on them if their opportunities were better than they are". With that view, we may think of the poor as referring to the rich as not being in poverty, and thus to their incomes as not being below the poverty line, that is, as being equal to $z$. This avoids comparisons of the poor with some potentially very large incomes, which the poor may consider as irrelevant to establishing their relative deprivation as poor persons.

As noted above, the concept of relative deprivation is linked to the current widespread concern for social exclusion, which, as Silver (1994, p.557) remarks, entails "the drawing of inappropriate group distinctions between free and equal individuals which deny access to or participation in exchange or interaction", including participation in the socially perceived minimum consumption level. When $v=1$, no account is taken of relative deprivation in the computation of the poverty index. The higher the value of $v$, the more important is relative deprivation in assessing poverty, and the more important is the relative deprivation of the most excluded in assessing overall relative deprivation.

$v$ can then be usefully seen as an "exclusion-aversion" sensitivity parameter. $S(v)$ itself can be interpreted as a money-metric per capita normative cost of poverty, just as $I(v)$ can be seen as the mean-normalised per capita normative cost of inequality (see Atkinson (1970) and Sen (1973)). Since $\int_{0}^{1} \omega(p, v)=1$, it is indeed clear from (18) that a value of $S(v)$ for our poverty index can be thought of as being ethically equivalent to a situation in which all have a poverty gap equal to $S(v)$ :

$$
\int_{0}^{1} \omega(p, v) S(v) d p \equiv S(v) .
$$

$S(v)$ can thus be thought as the equally distributed equivalent (EDE) poverty gap that is assessed by an analyst when using a particular value of $v$. When $v=2$, this EDE poverty gap reduces to the Thon(1979)- Chakravarty(1983)Shorrocks(1995) index (itself much influenced by Sen's (1976) seminal index), which has been used for instance recently by Osberg and Xu (2000) and Myles and Picot (2000) to decompose changes in the $S(2)$ poverty index 
into changes in the average poverty gap and changes in the (standard Gini) inequality in censored incomes.

\subsection{Poverty and growth}

Poverty assessments and poverty profiles are often made to guide public policy analysis. We might thus wish to know by how much the $S(v)$ indices of poverty would fall if all incomes rose by one dollar (following, say, a uniform fall in a poll tax or an increase in a lump-sum transfer), or if all incomes increased by the same proportion (following, say, a surge in some inequality-neutral economic growth). These changes in poverty can in particular guide the design of subsidies or transfer targetting, in the manner of Besley and Kanbur (1988) for instance. For this purpose, we define $S_{0}(v)$ as the $S(v)$ index when all of the poor in a distribution are assumed to have zero incomes. It is possible to show that $S_{0}(v)=z\left[1-(1-H)^{v}\right]$. For a uniform per capita marginal income change, $d \gamma$, we then find that

$$
\frac{d S(v)}{d \gamma}=(1-H)^{v}-1=-\frac{S_{0}(v)}{z}
$$

Equation (23) is straightforward to compute since it only requires the headcount, the poverty line and the ethical parameter $v$. The greater the focus on the poorest (when $v$ is large), the greater the change in deprivation since the increase in $\gamma$ is then deemed to be more effective. The increase in income for those above the poverty line has indeed no effect on deprivation, absolute or relative, and this is seen as wasted when relative deprivation and ethical focus on the poorest are given little weight in assessing poverty.

For a proportional marginal change $d \lambda$ of all incomes, we find that:

$$
\frac{d S(v)}{d \lambda}=S(v)-S_{0}(v) .
$$

whose computation again only requires knowledge of $v$, the headcount and the pre-change poverty index. Hence, a $1 \%$ inequality-preserving increase in GNP reduces poverty most when "maximum poverty" $S_{0}(v)$ is large compared to $S(v)$. This corresponds to a situation where the poor are many but absolutely and relatively little deprived, namely, to a situation where inequality is not too strong an impediment to poverty alleviation through equiproportional economic growth (on this, see for instance the recent papers by Ravallion (1997) and De Janvry and Sadoulet (2000)). 


\subsection{Subgroup decomposition}

Although the $S(v)$ indices have a nice graphical interpretation and have been shown to be a sum of absolute and relative deprivation, they are not subgroup decomposable in the sense of Foster and Shorrocks (1991), since they cannot be expressed as a sum of poverty indices defined separably over exclusive and exhaustive subgroups. Since $S(v)$ can be expressed as an integral of weighted incomes, we will see, however, that it is straightforward to decompose overall poverty as a sum of subgroup contributions, with the contributions involving individual weights that depend on the rank of individuals in the overall distribution of income. It is this dependence on ranks in the overall distribution that makes the $S(v)$ indices not decomposable in the sense indicated above.

The property of separability is not, however, as desirable as is sometimes suggested in the literature. It is unlikely for instance that in comparing themselves with others, individuals confine themselves to tight socio-economic groups. Instead, if concerns of relativity ought to scan the whole distribution of income to be relevant for the measurement of poverty, then separability is clearly not a desirable property for a poverty index. Hence, we would not wish a change in the distribution of incomes in a group to leave poverty unaltered in another group if assessments of relative deprivation must be made taking into account the whole population, and not a single subgroup. Or, to paraphase Sen $(1973, \mathrm{p} .41)$, "if one feels that the social valuation of the welfare of individuals should depend crucially on the levels of welfare (or incomes) of others, this property of the independence of each person's welfare component from the position of others [in other subgroups] has to be sacrificed."

To see how to decompose $S(v)$ into subgroup components, denote by $M$ the number of subgroups, define as $\Pi_{m}(p)$ the density of being a member of group $m$ at population percentile $p$, with $\sum_{m=1}^{M} \Pi_{m}(p)=1$, and define $G_{m}(p)$ as

$$
G_{m}(p)=\frac{1}{\Pi_{m}} \int_{0}^{p} \Pi_{m}(s) g(s) d s
$$

where $\Pi_{m}=\int_{0}^{1} \Pi_{m}(p) d p$ is the proportion of group $m$ members in the population. $G_{m}(p)$ thus cumulates the poverty gaps of members of group $m$ up to population rank $p$. We then have that

$$
G(p)=\sum_{m=1}^{M} \Pi_{m}(p) G_{m}(p) .
$$


Defining the poverty index $S_{m}(v)$ for group $m$ as

$$
S_{m}(v)=\int_{0}^{1} k(p, v) G_{m}(p) d p
$$

we easily find that :

$$
S(v)=\sum_{m=1}^{M} \Pi_{m} S_{m}(v) .
$$

\subsection{Comparison of the properties of the $S(v)$ indices with those of additive indices}

The Foster, Greer and Thorbecke (1984) class of poverty indices has become in the last two decades the most popular class of poverty indices used in theoretical and empirical studies of poverty. The FGT indices are defined as:

$$
F G T(\alpha)=\int_{0}^{1} g(p)^{\alpha} d p
$$

where $\alpha$ is a non-negative parameter of ethical aversion to "inequality in poverty gaps". $F G T(\alpha=0)$ gives the headcount index. $F G T(\alpha=1)$ is the average poverty gap (that is, $H I$ or $S(v=1)$ ). For larger values of $\alpha$, the FGT index is an average of some power of the poverty gaps. The larger the value of $\alpha$, the greater the ethical weight given to larger poverty gaps in measuring and comparing poverty.

The perceived and oft-mentioned advantages of the FGT class of indices are its ethical flexibility (captured by the parameter $\alpha$ ), its decomposability across subgroups ${ }^{8}$, and its simplicity of computation and understanding. Although we are not presumptuous enough (!) to believe that this paper will (or in fact should) alter this popularity, we believe that the properties of the $S(v)$ indices compare rather well with those of the FGT additive indices. We review and compare some of these properties now.

1) The $S(v)$ indices are not subgroup-decomposable. As argued above in Section 3.3, decomposability across subgroups is, however, not necessarily a desirable property. This argument is reminiscent of the perceived desirability/undesirability of subgroup decomposability in the

\footnotetext{
${ }^{8}$ Note that other decomposable indices include the Clark, Hemming and Ulph (1981), Chakravarty (1983), and Watts (1968) indices.
} 
literature on inequality measurement, reflected for instance in the debate between the proponents of the classes of generalised entropy indices and of linear indices of inequality (including the Gini). Section 3.3 nevertheless suggested how we may show graphically the contribution of different subgroups to the cumulative poverty gap curve $G(p)$ at various values of $p$, and thus how some decomposition of total poverty across subgroups could be obtained mainly for illustrative purposes.

2) One of the frequent complaints made about the Gini index until the work of Donaldson and Weymark (1980, 1983) and Yitzhaki (1983) was its ethical rigidity. Building on that latter work, the single-parametrisation of the class $S(v)$ of poverty indices makes it as ethically flexible as the class of FGT indices. For both the FGT and the $S(v)$ indices, this flexibility allows the analyst to incorporate in poverty comparisons greater or lesser weight to inequality in well-being. For the $S(v)$ indices, ethical flexibility has the particular advantage of being interpretable as flexibility on the weight granted to individual relative deprivation in assessing total deprivation, and more particularly on the weight given to the relative deprivation of the most deprived.

3) $S(v)$ is easily interpreted as a weighted average of poverty gaps in a distribution of well-being. As indicated above in Section 3.1, it is also the equally distributed poverty gap that is socially equivalent to the actual distribution of poverty gaps. Whatever the value of $v, S(v)$ is thus money-metric, and is also easily interpreted as the socially representative deprivation in a population, a feature which is not shared by the FGT indices for $\alpha$ different from $1^{9}$.

4) Since the CPG curve was shown in earlier work to have a nice role in testing poverty dominance (apart from having nice graphical features in itself), its use in poverty analysis is certain to become important in the future. This makes the use of $S(v)$ attractive, since it has a nice geometric interpretation in terms of a weighted area underneath the CPG curve. This interpretation holds for any value of $v$. Furthermore, the inequality component for the $S(v)$ indices has a nice conceptual and graphical interpretation in terms of a sum of individual relative deprivation along the $p$ values.

\footnotetext{
${ }^{9} \mathrm{~A}$ money-metric index that is ordinally equivalent to the FGT index can be obtained simply by using $(F G T(\alpha))^{1 / \alpha}$, but this transformation of the FGT indices would cost them their popular additivity property.
} 


\section{An illustration using LIS data}

To illustrate some of the above relations, we use data drawn from the Luxembourg Income Study (LIS) ${ }^{10}$ data sets of Belgium and Denmark (1992 data) and of Italy and the USA (1991 data). These two pairs of countries were partly selected because of the interesting features they exhibit in poverty comparisons, as will become clearer later. The raw data were treated in the same manner as in Gottschalk and Smeeding (1997), and yielded household disposable income (i.e., post-tax-and-transfer income) expressed in 1991 adult-equivalent $\$$ US ${ }^{11}$. The reference poverty line was set at $\$ 7000$ in 1991 adult-equivalent US dollars, which appeared to be a reasonable baseline for poverty comparisons across industrialised countries, and which is also approximately the 1991 American poverty line for single individuals. 12 Finally, since the results here are purely illustrative, we do not present here standard errors for our various estimates (they can be ontained from the authors upon request), although it will be clear from inspection that some of the cross-country comparisons discussed below are not statistically significant $^{13}$.

Table 1 shows the headcounts for the 4 countries mentioned above at poverty lines of US $\$ 7000$ and slightly above. Italy has by far the most poverty by this standard, followed by the United States, Belgium and Denmark. The first column of Table 2 shows the $S(1)$ values for the same countries at $z=\$ 7000$, which is simply the average poverty gap $H I$. Unlike the poverty headcounts, the average poverty gaps (and thus absolute deprivation) are very similar in Belgium and in Denmark, and in Italy and in the USA respectively. It will thus be interesting to check if relative deprivation is

\footnotetext{
${ }^{10}$ See http://lissy.ceps.lu for detailed information on the structure of these data.

${ }^{11}$ We apply purchasing power parities drawn from the Penn World Tables (see Summers and Heston (1991) for the methodology underlying the computation of these parities, and http://www.nber.org/pwt56.html for access to the 1991 figures) to convert national currencies into 1991 US dollars. As in Gottschalk and Smeeding (1997), we divide household income by an adultequivalence scale defined as $h^{0.5}$, where $h$ is household size, so as to allow comparisons of the welfare of individuals living in households of different sizes. Hence, all incomes are transformed into 1991 adult-equivalent \$US. All household observations are also weighted by the LIS sample weights "hweight" times the number of persons in the household. Finally, negative incomes are set to 0 .

${ }^{12}$ This poverty line is precisely equal to US\$7086. We thank Buhong Zheng for this information.

${ }^{13}$ The standard errors can be computed from the results of Theorem 4 in Davidson and Duclos (2000), which shows the asymptotic sampling distribution of CPG curves. These formulae and others have been programmed by Duclos, Araar and Fortin (2000) in the software DAD (Distributive Analysis/Analyse distributive) which is freely available at www.mimap.ecn.ulaval.ca.
} 
sufficiently different across these countries to affect cross-country comparisons. Figure 3 shows how individual relative deprivations $c(p)$ vary across different quantiles $p$ for each of the four countries. The United States show more relative deprivation than Belgium and Denmark whatever the quantiles considered. The Italian relative deprivation profile crosses that of the four other countries. This also says that although mean absolute deprivation is substantially greater in Italy than in Denmark or in Belgium, for individuals towards the bottom of the income distributions, relative deprivation does not differ by much (and can in fact be greater in Denmark than in Italy). The $c(p)$ curve for Italy crosses that of the US at around $p=0.09$; looking at equation (15), comparisons of the inequality in poverty gaps across Italy and the United States can thus be expected to be ambiguous and to depend on the ethical parameter $v$.

Before aggregating absolute and relative deprivation, it is useful to consider the CPG curves for the four countries. Figure 4 does this. Multiple crossings of the CPG curves occur, and only one unambiguous sample ordering can be made in the 6 possible comparisons of countries (for inference of population orderings, we would need to take into account sampling variability). Since the sample CPG curve for Denmark is everywhere below that for the US, it is possible to say that poverty is unambiguously greater for the US sample than for Denmark for all of the poverty indices $\pi \in \Pi$ discussed in (4). The CPG curve for Belgium crosses twice the CPG curve of Denmark, and the Italian CPG curve crosses the US curve from below at the very end.

One way to assess the ethical sensitivity of the poverty comparisons is to compute the $S(v)$ indices for various values of the ethical parameter $v$. This is shown in Table 2, with aggregate relative deprivation indicated in parentheses. For $v$ equal to 2,3 and 4, poverty is lower in Belgium than in Denmark, Italy or the United States, and Danish poverty is lower than in Italy and the United States (as was expected from the ranking of the CPG curves). The comparisons of Italian and American poverty depend on $v$ and thus on the importance given to relative deprivation in measuring poverty. For the headcount and for absolute deprivation, Italy has more poverty than the US, but when sufficient weight is given to relative deprivation (for $v \geq 2$ for instance), poverty in the US becomes significantly greater.

Figures 5 and 6 show graphically how the indices change with variations in $v$ and marginal changes in $z$. Figure 5 confirms that at a poverty line of $\$ 7000$, Denmark always has more poverty than Belgium, whatever the value of $v$, since it has both more absolute deprivation and generally more individual relative deprivation whatever the percentile considered (recall Figure 
3 ). When the poverty line increases up to $\$ 7500$, however, Belgium starts to have higher absolute deprivation, and it is then only with suitably high weights on the relative deprivation of the poor that Belgian poverty can still be considered lower than the Danish one. Similar remarks apply to the comparison of poverty between Italy and the US in Figure 6 . For $z \geq \$ 7000$, Italian poverty can be considered greater than American poverty only when sufficiently low weight is given to the importance of relative deprivation in measuring poverty. Otherwise, Italy has less poverty than the US.

Finally, Table 3 shows how poverty in the four countries responds either to a $\$ 1$ increase $\left(\frac{d S(v)}{d \gamma}\right)$ or to an equiproportionate increase $\left(\frac{d S(v)}{d \lambda}\right)$ in everyone's income. As equations (23) and (24) show, these responses depend on the importance $v$ given to concerns of relative deprivation, on the population proportion of the poor and on whether the poor are in deep or in shallow deprivation. The greater the focus on relative deprivation, the more sensitive the $S(v)$ indices are to equal absolute changes in incomes; the more numerous the poor, the greater the sensitivity of the $S(v)$ indices to equal absolute changes in incomes; and the deeper the absolute and relative deprivation of the poor, the less responsive are the $S(v)$ indices to equal equiproportionate changes in everyone's incomes.

As expected, we find in Table 3 that increases in $v$ and in the focus granted to relative deprivation increase the reaction of poverty to absolute and equiproportional growth in incomes. For instance, a $\$ 1$ increase in everyone's income in Belgium will decrease $v(1)$ by 0.092 , but will bring $v(4)$ down by 0.320 . Table 3 also shows that although Table 2 reports numerically close $S(v)$ indices for Belgium and Denmark and for Italy and the United States, the reaction of these indices to changes in incomes are very different. Since Belgium has more poor than Denmark, its poverty indices react much more strongly to equal increases of $\$ 1$, and so does Italy when compared to the United States. As for a $1 \%$ growth in everyone's income, it is estimated to bring poverty down much faster in Belgium than in Denmark, and almost twice as quickly for Italy as for the United States. Because the $S(v)$ indices (including $v(1)$, the average poverty gap) are close within these two pairs of countries, these important differences are explained by the depth and the concentration of the relative deprivation experienced by the poor. Deprivation in the US is concentrated on a smaller proportion of the population than in Italy (see Figure 3); it is thus also more deeply and more relatively felt by the poorest. This makes inter alia inequality-neutral economic growth much less effective in the United States than in Italy as an instrument of poverty reduction. 


\section{Conclusion}

Our paper develops the link between poverty and inequality by focussing on a class of poverty indices which aggregate concerns of absolute deprivation and relative deprivation. The indices depend upon an ethical parameter $v$ which captures the ethical sensitivity of poverty measurement to "exclusion" or "relative-deprivation" aversion. We show that the indices equal the sum of mean absolute deprivation and of an ethically weighted mean of the individual relative deprivation found among the poor. The greater the value of $v$, the greater the weight assigned to relative deprivation as against absolute deprivation in measuring and comparing poverty. We also show how the indices can be easily used to assess the impact of growth on poverty, and compare some of their properties to those of the popular class of FGT indices.

Our illustrative section reports that, for a reasonable common poverty line, the United States have more relative deprivation than Denmark and Belgium whatever the percentiles considered. For comparisons of total deprivation, however, it is not possible to order these countries robustly. Since absolute deprivation is very similar in the four countries considered, poverty comparisons across them will inevitably depend on the importance granted to concerns over relative deprivation. The impact of growth on poverty is also seen to depend on the presence of and on concerns over relative deprivation: in pairwise comparisons of Italy and the US and of Belgium and Denmark, poverty is much less responsive to growth in the USA and in Denmark, which is also where relative deprivation is generally found to be the greatest.

\section{Appendix}

We show here the derivation of equations (11), (14) and (15). First note that

$$
\begin{aligned}
\int_{0}^{p} k(s, v) d s & =\int_{0}^{1} k(s, v) d s-\int_{p}^{1} k(s, v) d s \\
& =v-\omega(p, v)
\end{aligned}
$$

Integrating by parts $I(v)$ (as defined by (9)) then yields:

$$
I(v)=\int_{0}^{1}(p-L(p)) k(p, v) d p
$$




$$
\begin{aligned}
& =\left.(v-\omega(p, v))(p-L(p))\right|_{0} ^{1}-\int_{0}^{1}(v-\omega(p, v))\left(1-\frac{y(p)}{\mu}\right) d p \\
& =\int_{0}^{1} \omega(p, v)\left(1-\frac{y(p)}{\mu}\right) d p
\end{aligned}
$$

which is also equation (11). Equation (14) is obtained by noting that:

$$
\begin{aligned}
c\left(p_{i}\right) & =\int_{p_{i}}^{1}\left(y(p)-y\left(p_{i}\right)\right) d p \\
& =\int_{0}^{1} y(p) d p-\int_{0}^{p_{i}} y(p) d p-\left(1-p_{i}\right) y\left(p_{i}\right) \\
& =\mu\left(1-L\left(p_{i}\right)\right)-\left(1-p_{i}\right) y\left(p_{i}\right) .
\end{aligned}
$$

To show equation (15), first note from the above and from the definition of $k(p, v)$ that

$$
\begin{aligned}
& \frac{1}{v \mu} \int_{0}^{1} c(p) k(p, v) d p= \\
& (v-1) \int_{0}^{1}\left((1-L(p))(1-p)^{(v-2)}-\frac{y(p)}{\mu}(1-p)^{v-1}\right) d p
\end{aligned}
$$

Integrating by parts $\frac{y(p)}{\mu}(1-p)^{(v-1)}$ by integrating $y(p) / \mu$ to yield $L(p)$ and differentiating $(1-p)^{(v-1)}$, we find:

$$
\frac{1}{v \mu} \int_{0}^{1} c(p) k(p, v) d p=1-\int_{0}^{1} L(p) k(p, v) d p .
$$

Since $\int_{0}^{1} p k(p, v) d p=1,(34)$ is the same as the definition of $I(v)$ in equation (9). 


\section{References}

[1] Atkinson, A.B. (1970). "On the Measurement of Inequality", Journal of Economic Theory, 2, 244-263.

[2] Atkinson, A.B. (1987). "On the Measurement of Poverty", Econometrica, 55, 749-764.

[3] Atkinson, A.B. (1991). "Measuring Poverty and Differences in Family Composition", Economica, 59, 1-16.

[4] Besley, Timothy J. and S.M. Ravi Kanbur (1988), "Food Subsidies and Poverty Alleviation", Economic Journal, 98, 701-719.

[5] Blackorby, C. and D. Donaldson (1978). "Measures of Relative Equality and Their Meaning in Terms of Social Welfare", Journal of Economic Theory, 18, 59-80.

[6] Blackorby, C. and D. Donaldson (1980). "Ethical Indices for the Measurement of Poverty", Econometrica, 48, 1053-1061.

[7] Chakravarty, S.R. (1983). "A New Index of Poverty" Mathematical Social Sciences, 6, 307-313.

[8] Chakravarty, S.R. (1990). Ethical Social Index Numbers, New York, Springer-Verlag.

[9] Clark, S., R. Hemming and D. Ulph (1981). "On Indices for the Measurement of Poverty", The Economic Journal, 91, 515-526.

[10] Davidson, R. and J.Y. Duclos (2000), "Statistical Inference for Stochastic Dominance and the for the Measurement of Poverty and Inequality", Econometrica, 68, 1435-1465.

[11] De Janvry, Alain, and Elisabeth Sadoulet (2000), "Growth, Poverty, and Inequality in Latin America: A Causal Analysis, 1970-94", Review of Income and Wealth, 46, 267-288.

[12] Donaldson, D. and J.A. Weymark (1980). "A Single Parameter Generalization of the Gini Indices of Inequality", Journal of Economic Theory, 22, 67-86.

[13] Duclos, J.-Y. (1999) "Gini Indices and the Redistribution of Income", International Tax and Public Finance, 7, 141-162.

[14] Duclos, J.-Y., Araar, A. and C. Fortin (2000), "DAD: A Software for Distributive Analysis / Analyse distributive", MIMAP, International Research Centre, Government of Canada and CRÉFA, Université Laval (www.mimap.ecn.ulaval.ca). 
[15] Durant, T.J. and O. Christian (1990), "Socio-Economic Predictors of Alienation Among the Elderly", International Journal of Aging and Human Development, 31, 205-217.

[16] Foster, J.E., (1984). “On Economic Poverty: A Survey of Aggregate Measures", in R.L. Basmann and G.F. Rhodes, eds., Advances in Econometrics, 3, Connecticut, JAI Press, 215-251.

[17] Foster, J., J. Greer and E. Thorbecke (1984). "A Class of Decomposable Poverty Measures", Econometrica, 52, 761-767;

[18] Foster, J.E. and A. Sen (1997). "On Economic Inequality after a Quarter Century", in On Economic Inequality, (expanded edition), Oxford: Clarendon Press.

[19] Foster, J.E. and A.F. Shorrocks (1988). "Inequality and Poverty Orderings" European Economic Review, 32, 654-662.

[20] Foster, J.E. and A.F. Shorrocks (1991). "Subgroup Consistent Poverty Indices”, Econometrica, 59, 687-709.

[21] Gottschalk, P. and T.M. Smeeding (1997). "Cross-National Comparisons of Earnings and Income Inequality" Journal of Economic Literature, 35, 633-687.

[22] Hagenaars, A. (1987). "A class of poverty indices", International Economic Review, 28 583-607.

[23] Hey, J.D. and P.J. Lambert (1980), "Relative Deprivation and the Gini Coefficient: Comment", Quarterly Journal of Economics, 95,567-573.

[24] Jenkins, S.P. and P.J. Lambert (1997). "Three 'I's of Poverty Curves, With an Analysis of UK Poverty Trends" Oxford Economic Papers, 49, 317-327.

[25] Kakwani, N. (1980). "On a Class of Poverty Measures", Econometrica, 48, 437-446.

[26] Lambert, P. (1993). The Distribution and Redistribution of Income: a Mathematical Analysis, 2nd edition, Manchester University Press, Manchester.

[27] Mehran, F. (1976) "Linear Measures of Income Inequality", Econometrica, 44, 805-809.

[28] Muliere, P. and M. Scarsini (1989), "A Note on Stochastic Dominance and Inequality Measures", Journal of Economic Theory, 49, 314-323.

[29] Myles, J. and G. Picot (2000), "Poverty indices and policy analysis", The Review of Income and Wealth, 46, 161-180. 
[30] Olson, J.M., P. Herman and M.P. Zanna (eds.), (1986), Relative Deprivation and Social Comparisons: The Ontario Symposium, vol.4, London, Lawrence Erlbaum Associates Publishers.

[31] Osberg, L. and K. Xu (2000), "International comparisons of poverty intensity: Index decomposition and bootstrap inference", Journal of Human Resources, forthcoming.

[32] Ravallion, M. (1997). "Can high-inequality developing countries escape absolute poverty?", Economics Letters, 56, 51-57.

[33] Room, G. et al. (1992), Second Annual Report, Observatory on National Policies to Combat Social Exclusion, Directorate General for Employment, Social Affairs and Industrial Relations, Commission of the European Communities, Brussels.

[34] Runciman, W. G. (1966), Relative Deprivation and Social Justice: A Study of Attitudes to Social Inequality in Twentieth-Century England, Berkeley and Los Angeles, University of California Press.

[35] Sen, A.K. (1973), On Economic Inequality, Clarendon Press, Oxford.

[36] Sen, A. (1976). "Poverty: An Ordinal Approach to Measurement", Econometrica, 44, 219-232.

[37] Sen, A., (1981). Poverty and Famine: An Essay on Entitlement and Deprivation, Clarendon Press, Oxford University Press.

[38] Shorrocks, A., F. (1995). "Revisiting the Sen Poverty Index", Econometrica, 63, 1225-123.

[39] Shorrocks, A.F. (1998), "Deprivation Profiles and Deprivation Indices" ch.11 in The Distribution of Household Welfare and Household Production, ed. S. Jenkins et al., Cambridge University Press.

[40] Silver, H. (1994), "Social Exclusion and Social Solidarity: Three Paradigms", International Labour Review, 133, 531-576.

[41] Spencer, B.D. and S. Fisher (1992), "On Comparing Distributions of Poverty Gaps", The Indian Journal of Statistics, 54, Series B, Pt. 1, 114-126.

[42] Summers, R. and A. Heston (1991). "The Penn World Table (Mark 5): An Expanded Set of International Comparisons, 1950-1988" The Quarterly Journal of Economics, 106, 327-368.

[43] Takayama, N. (1979). "Poverty, Income Inequality, and Their Measure: Professor Sen's Axiomatic Approach Reconsidered", Econometrica, 47, 747-759. 
[44] Thon, D. (1979). "On Measuring Poverty", Review of Income and Wealth, 25, 429-439.

[45] Townsend, Peter (1979),Poverty in the United Kingdom : A Survey of Household Resources and Standards of Living, Berkeley, University of California Press.

[46] Watts, H.W. (1968). "An Economic Definition of Poverty", in D.P. Moynihan (ed.), On Understanding Poverty, New York: Basic Books.

[47] Yaari, Menahem E. (1988), "A Controversial Proposal Concerning Inequality Measurement”, Journal of Economic Theory, 44, 381-97.

[48] Yitzhaki, S. (1979), "Relative Deprivation and the Gini Coefficient", Quarterly Journal of Economics,93, 321-324.

[49] Yitzhaki, S., (1983), "On an Extension of the Gini Index”, International Economic Review, 24, 617-628.

[50] Zheng, Buhong (1997). “Aggregate Poverty Measures” Journal of Economic Surveys, 11, 123-63. 
Table 1: Headcounts for different poverty lines

\begin{tabular}{|c|c|c|c|c|}
\hline$z$ & $H_{B E}$ & $H_{D K}$ & $H_{I T}$ & $H_{U S}$ \\
\hline 7000 & 0.09186 & 0.06965 & 0.20478 & 0.13738 \\
\hline 7100 & 0.09601 & 0.074192 & 0.21038 & 0.14174 \\
\hline 7200 & 0.09886 & 0.077878 & 0.21678 & 0.14565 \\
\hline 7300 & 0.10702 & 0.081991 & 0.22384 & 0.14881 \\
\hline 7400 & 0.10891 & 0.086221 & 0.23113 & 0.15187 \\
\hline 7500 & 0.11756 & 0.090567 & 0.23781 & 0.15594 \\
\hline
\end{tabular}

Table 2: $S(v)$ and relative deprivation when $z=U S \$ 7,000$

\begin{tabular}{|l|c|c|c|c|}
\hline Country(year) & $S(1)$ & $S(2)$ & $S(3)$ & $S(4)$ \\
\hline Belgium(92) & 176.20 & 344.71 & 506.02 & 660.55 \\
& $(0)$ & $(168.51)$ & $(329.82)$ & $(484.35)$ \\
\hline Denmark(92) & 181.12 & 355.80 & 524.32 & 686.94 \\
& $(0)$ & $(174.68)$ & $(343.20)$ & $(505.82)$ \\
\hline Italy(91) & 350.35 & 661.79 & 939.85 & 1189.16 \\
& $(0)$ & $(311.44)$ & $(589.50$ & $(838.81)$ \\
\hline Usa(91) & 348.97 & 669.75 & 965.13 & 1237.60 \\
& $(0)$ & $(320.78)$ & $(616.16)$ & $(888.63)$ \\
\hline
\end{tabular}

Table 3: $\frac{d S(v)}{d \gamma}$ and $\frac{d S(v)}{d \lambda}$ when $z=U S \$ 7,000$

\begin{tabular}{|l|c|c|c|c|}
\hline Country(year) & $v=1$ & $v=2$ & $v=3$ & $v=4$ \\
\hline Belgium(92) & -0.092 & -0.175 & -0.251 & -0.320 \\
& -466.79 & -882.26 & -1251.36 & -1578.58 \\
\hline Denmark(92) & -0.070 & -0.134 & -0.195 & -0.251 \\
& -306.44 & -585.39 & -838.91 & -1068.95 \\
\hline Italy(91) & -0.205 & -0.368 & -0.497 & -0.600 \\
& -1083.11 & -1911.64 & -2540.17 & -3011.84 \\
\hline Usa(91) & -0.137 & -0.256 & -0.358 & -0.446 \\
& -612.68 & -1121.44 & -1541.64 & -1886.45 \\
\hline
\end{tabular}


Figure 1: Cumulative Poverty Gap Curves

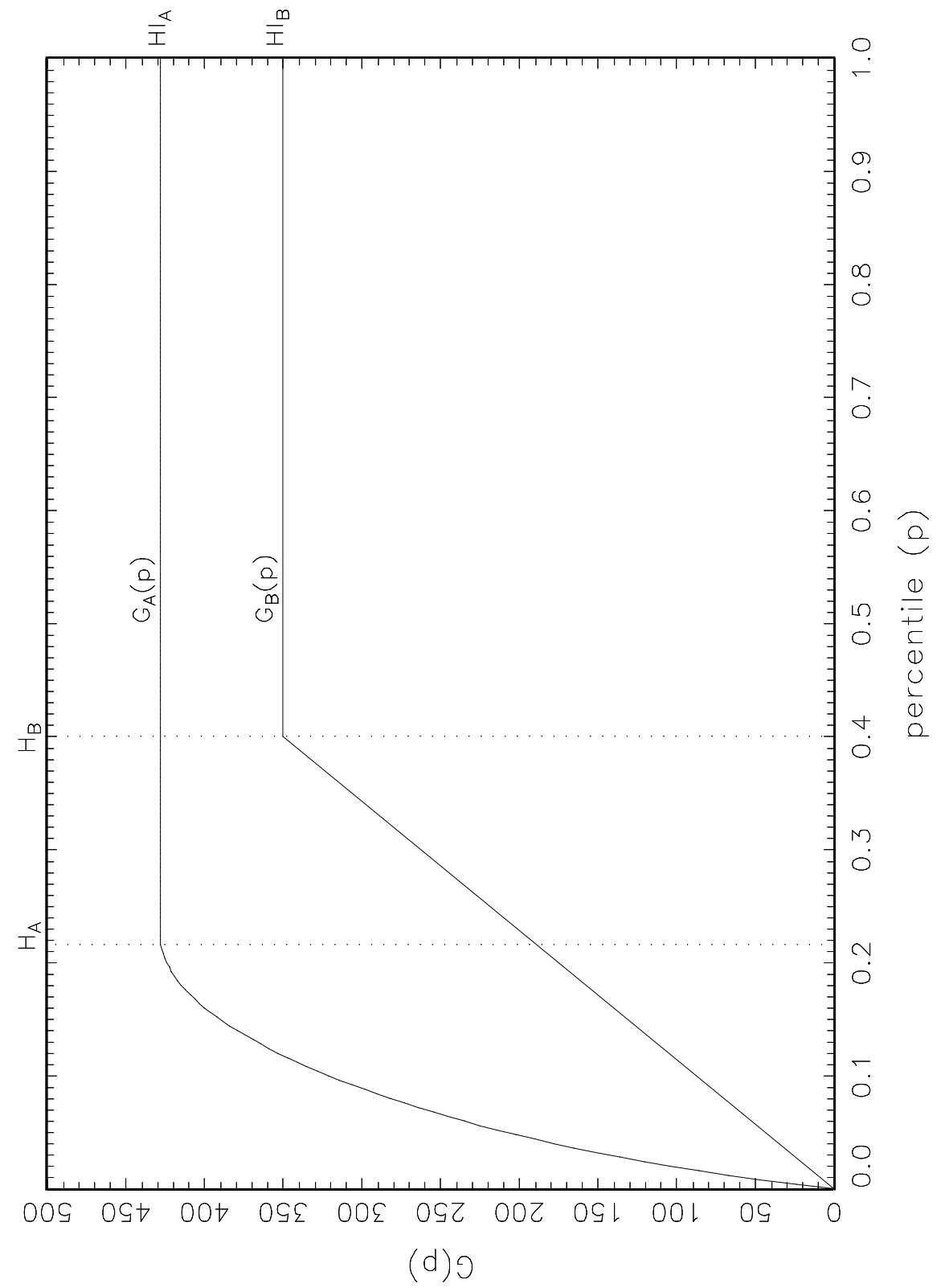


Figure 2: Absolute deprivation and inequality of poverty

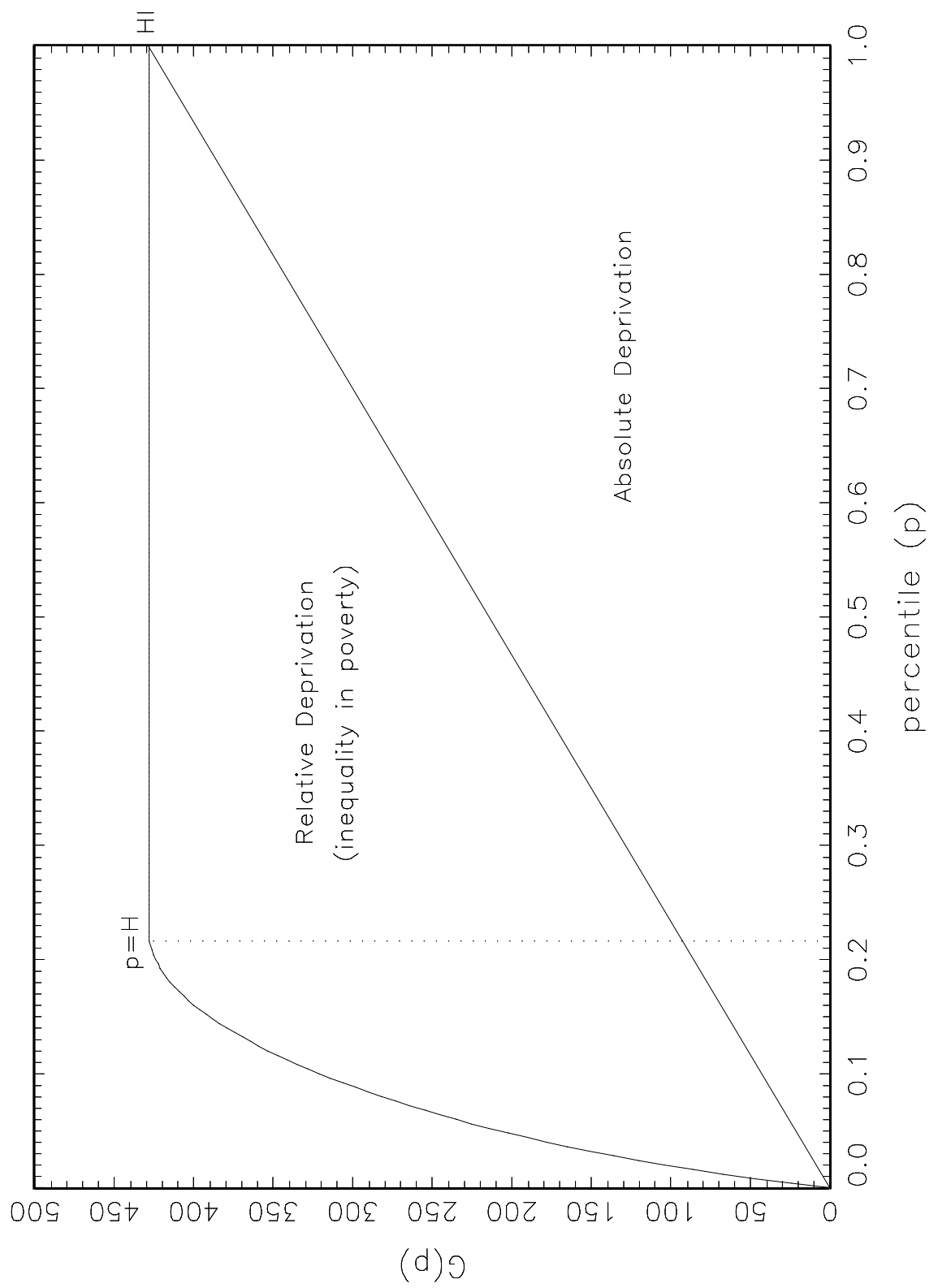


Figure 3: Relative Deprivation in Belgium, Denmark, Italy and the US $(z=$ $\$ 7000$ )

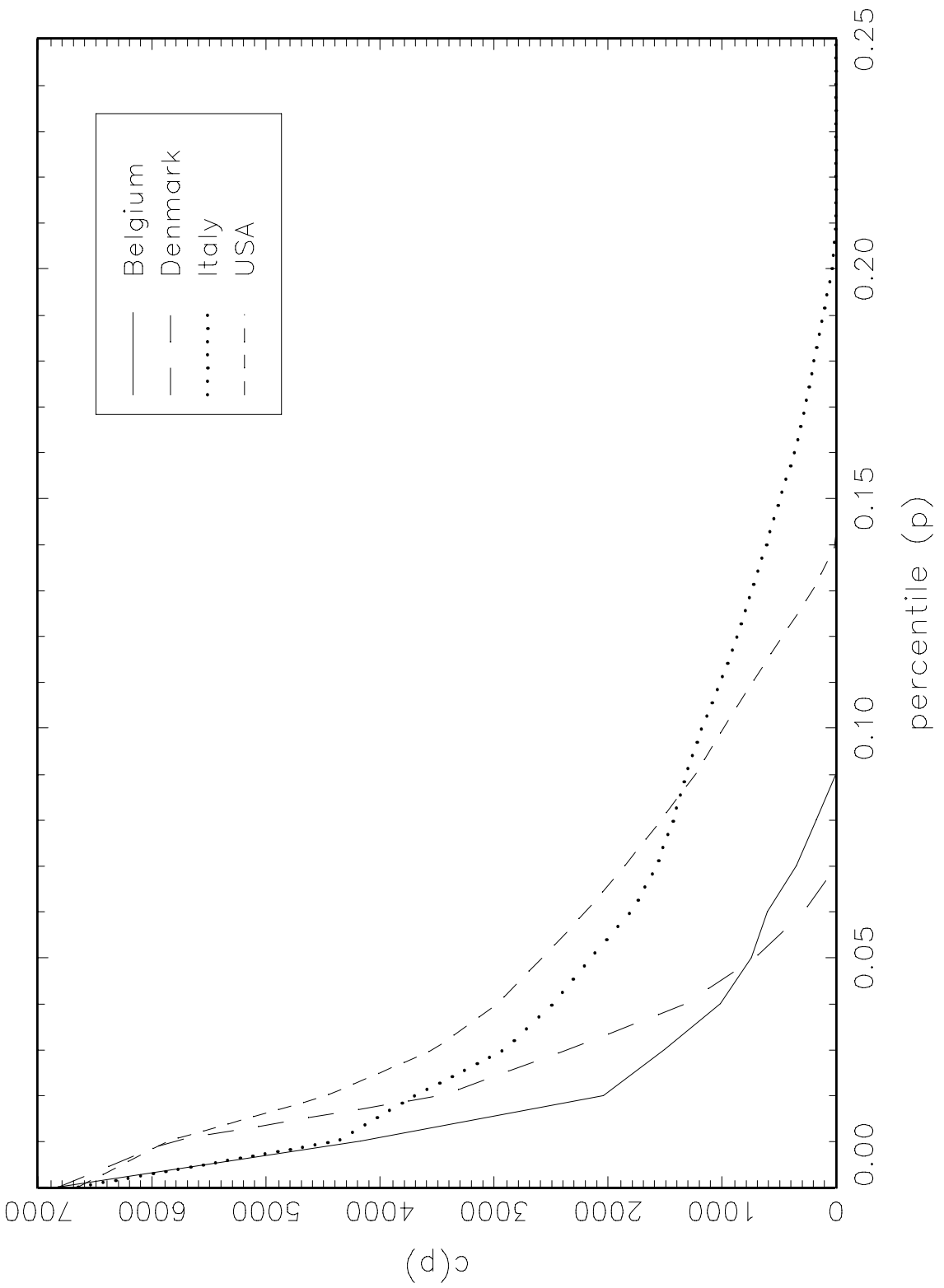


Figure 4: CPG curves for Belgium, Denmark, Italy and the US ( $\mathrm{z}=\$ 7000)$

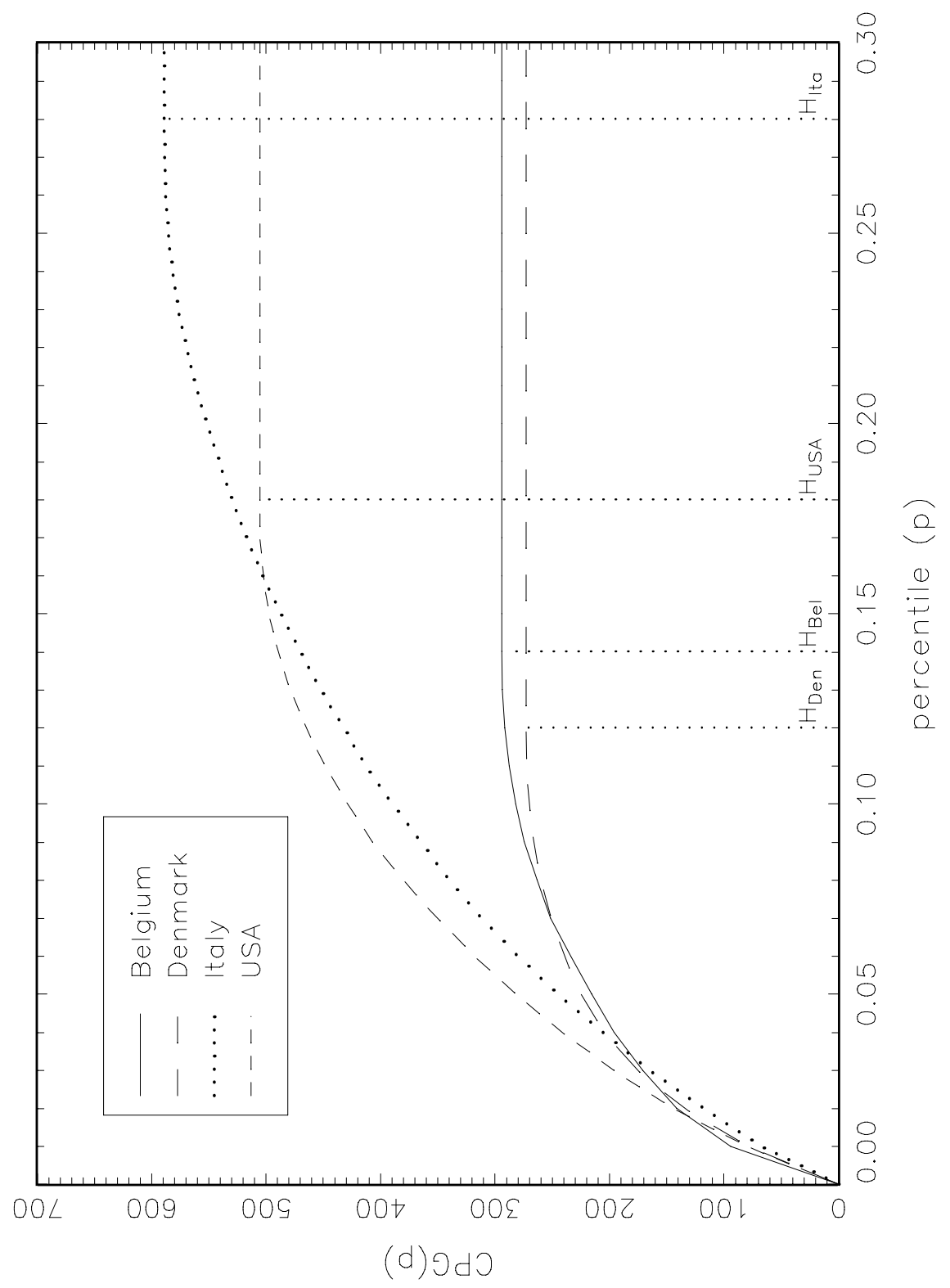


Figure 5: Difference between the Belgian and the Danish $S(v)$ indices for different $v$ and $z$

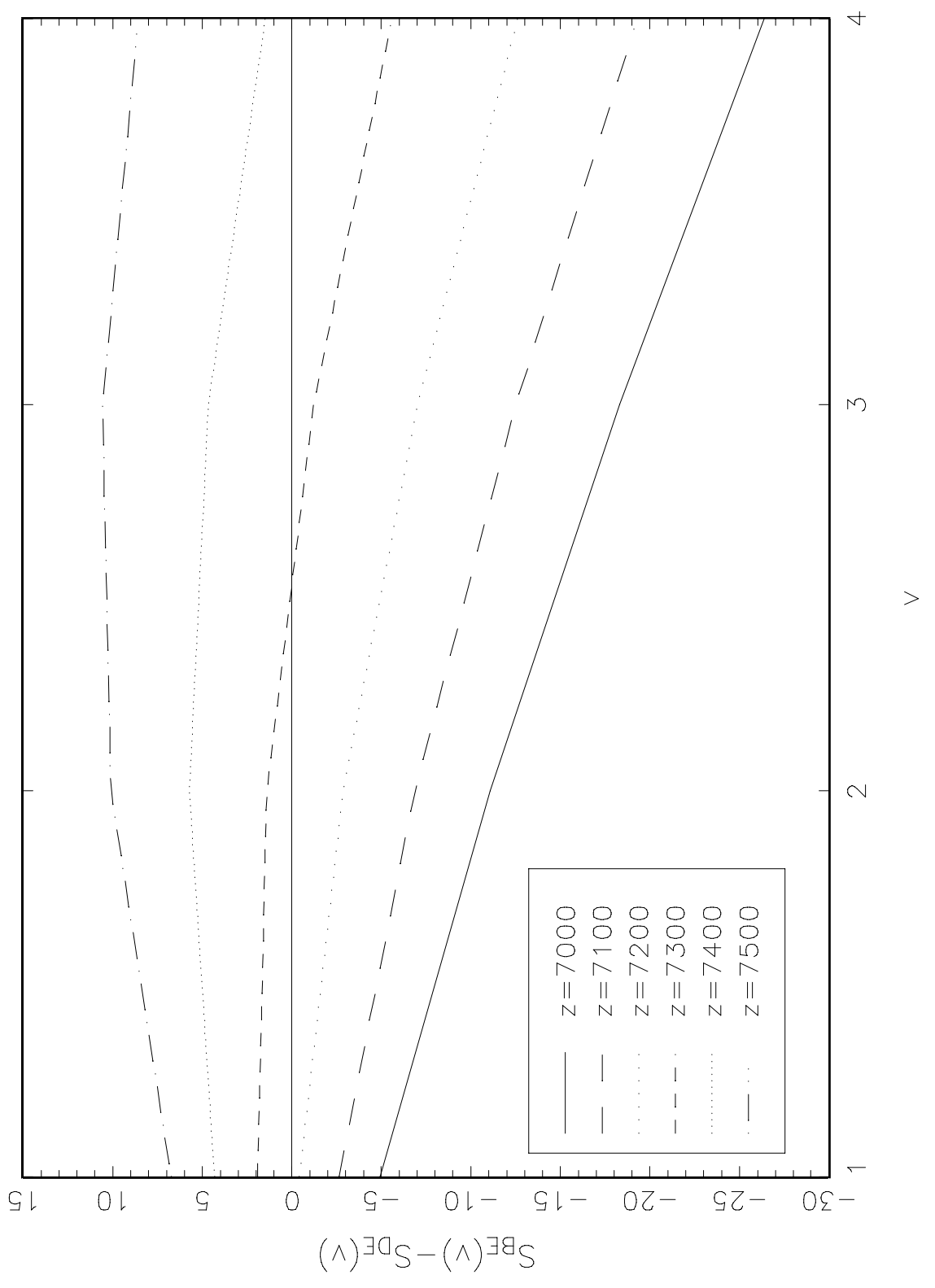

28 
Figure 6: Differences between the Italian and the American $S(v)$ indices for different $v$ and $z$

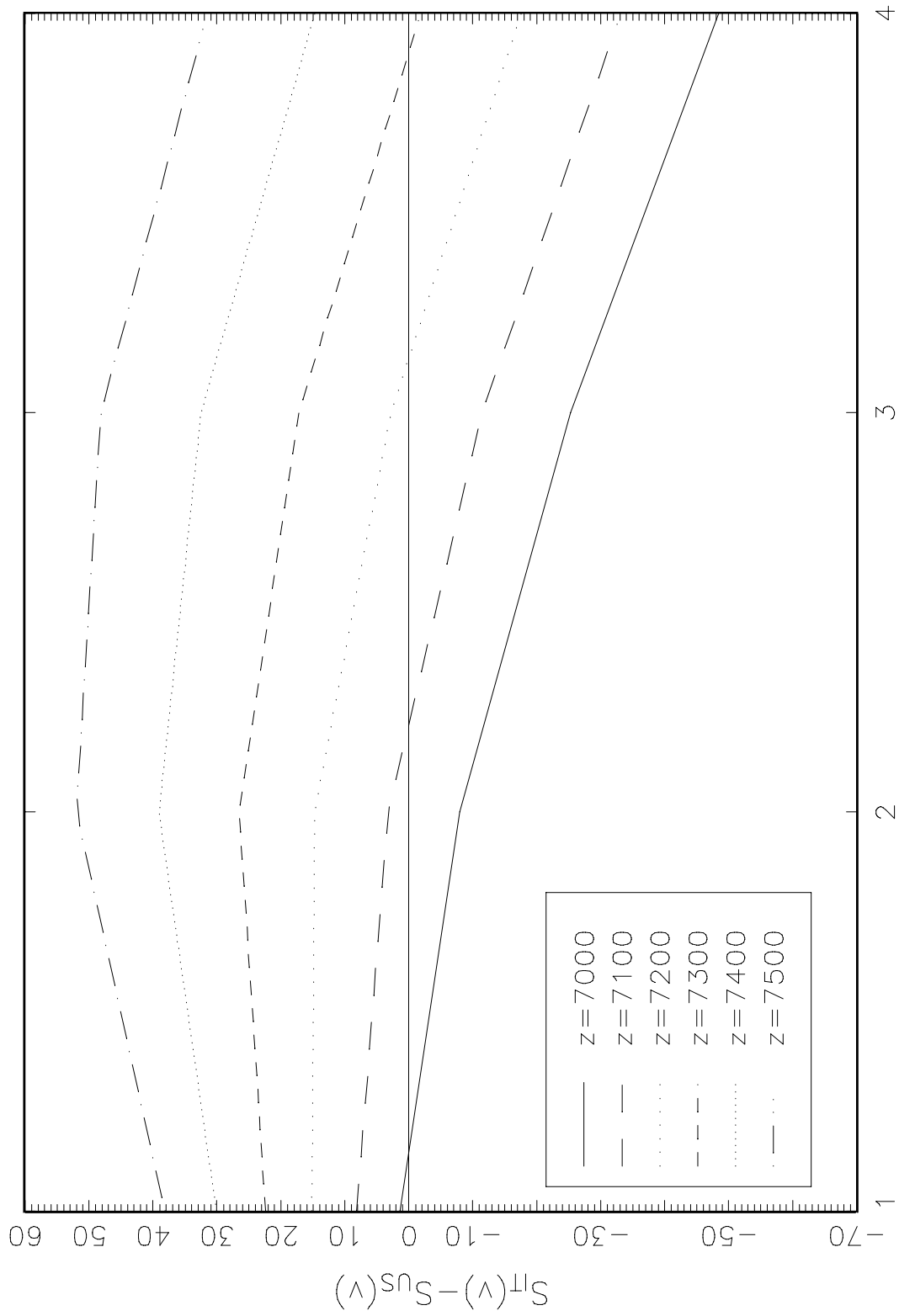

29 